\title{
EVALUATION OF BULK MATERIAL BEHAVIOR CONTROL METHOD IN TECHNOLOGICAL UNITS USING DEM. Part 2
}

\author{
A. V. Boikov ${ }^{1}$ R. V. Savelev1, V. A. Payor ${ }^{1}$, A. V. Potapov² \\ 1 St. Petersburg Mining University (St. Petersburg, Russia) \\ 2 ESSS (Florianopolis, Brazil)
}

E-mail: boikov_av@mail.ru

\section{AUTHOR'S INFO}

A.V. Boikov, Ph.D., Assistant Professor, Dept. of Automation of Technological Processes and Production,

R.V. Savelev, Student,

V. A. Payor, Student,

A. V. Potapov, Dr. Eng., Technical Director, ESSS Rocky DEM Chief Technology Officer

Key words:

DEM-modeling; pelletizing drums; classification of motion modes; neural networks; RNN; LSTM; bulk materials.

\author{
A B S T R ACT
}

The research is dedicated to the development of special devices (capsules) that can be used to control the mining ore behavior in the technological unit in order to increase processes efficiency. In the first part of the article, the choice of the discrete element method for generating various particle trajectories in the unit (drum pelletizer) was substantiated. This part describes the specific technologies that were used to recognize the pelletizing mode. In particular, conversation of paths to sensor readings is implemented using the Matlab Sensor Fusion and Tracking Toolbox. The obtained readings were processed using two neural network classifiers (DNN and LSTM). As a result, stable models for recognizing the pelletizing modes of the unit were obtained. LSTM recognition accuracy is greater than DNN. The developed approach can be used to recognize the operating modes of other technological units. In addition, data on particles trajectories can be used to improve DEM models of technological processes. Future work consists of the capsule physical implementation and testing the recognition algorithm on a real unit.

\section{Introduction}

In the first part of the article about research, that is devoted to the development of a device for bulk material behaviour control (trajectories) inside the technological unit using (the drum pelletizer as an example), we discussed evaluation of the method for recognizing the pelletizing mode. It was proposed to simulate the movement of the ore in a drum pelletizer using the discrete element method (DEM). Simulation of 20 numerical experiments produced 10981 trajectories of various types (different pelletizing modes) $[1-4,21]$.

The general concept of controlling the bulk material behaviour was presented by the authors in [5] and consists in assessing the modes of movement using capsules that repeat and record the movement of material in the unit (Fig. 1).

This article proposes a specific implementation of the method for converting the extracted data (trajectories) into the recognized pelletizing mode. The task of determining the pelletization mode (charge movement) by material accelerations (which are indirect parameters) relates to classification problems [6]. It is advisable to use machine learning methods, such as neural network classifiers to solve this class of problems. Neural network classifiers already successfully used for recognition of audio recordings, physical activity of a person, images, etc [7-8]. Neural network training requires a lot of data forming a dataset. As a rule, a dataset is formed on the basis of real observations and measurements accumulated over a rather large period of time. Since in this case we are only talking about the concept of the device, then to test

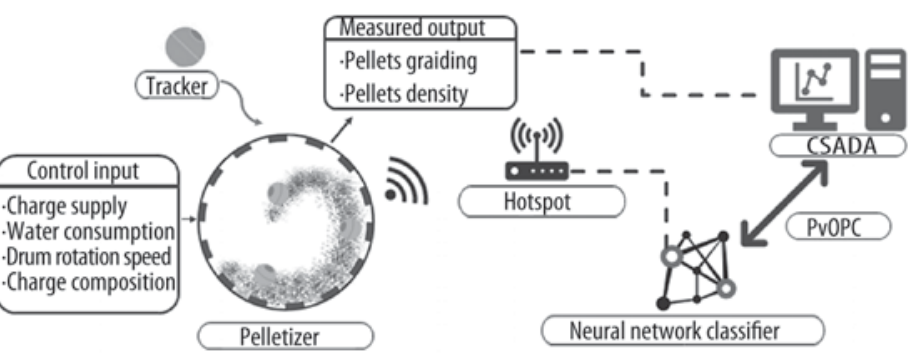

Fig. 1. The concept of using recording capsules in pelletizing drums [5]

the proposed solution, we will use the dataset obtained on a digital model of the technological process.

The data extracted from the simulation is an array of particle trajectories and their velocities (particles are used as an analog for capsules). However, the trajectories cannot be obtained in pure form on a real object. In accordance with the concept presented in [5] there was a need to converse the trajectories into the readings of the accelerometer, which is part of the inertial measuring module.

\section{Methodology}

\subsection{Convert paths to sensor readings}

Inertial measurement unit is an electronic device placed on the platform. IMU consists of a set of sensors, each of which transmits information about the movement of the platform. Typically, IMUs consist of combined sensors, including accelerometers, gyroscopes, and magnetometers [9]. The readings of the inertial measurement unit are not equivalent data obtained using DEM 
modeling. Thus, it is necessary to implement the translation of the numerical values of the particle motion paths into accelerations.

The special software Matlab Sensor Fusion and Tracking Toolbox [10] is used to convert the trajectories to sensor readings. This software package includes algorithms and tools for creating, simulating and analyzing various navigation systems and devices, including an inertial measurement unit. The behavior of an inertial measuring is simulated using the Inertial Sensor Fusion tool. In the Sensor Fusion and Tracking Toolbox software packages accelerations are obtained (based on the trajectories) and later used to recognize the modes of movement of granular media in the drum pelletizing.

\subsection{An algorithm for processing the obtained data to identify the movement modes of bulk material}

One of the possible options for implementing the data processing algorithm is the use of the trajectory elements recovery method which is based on an inertial navigation system [11]. Inertial navigation is basically determining the position of an object in space. Inertial navigation is based on Newtonian mechanics, the consonant of which is defined as the double integral of the acceleration [12] and accelerations are the source data received from the sensor.

However, the sequential integration of accelerations leads to the accumulation of errors and results in an increase of error in determining the coordinates of the body in proportion to the time the system [13].

Currently, there are many different methods for reducing error, one of which is ZUPT (Zero Velocity UPdaTe algorithm). It captures object complete stops or moments when object speed is close to zero and subsequently reset the initial conditions (reference point) [14].

Despite the fact that this method significantly reduces the error in determining the coordinates of an object in space, it is practically not applicable in industrial units, since the movement of granular medium in pelletizing drums is continuous. That is, the material practically does not stop, but only slightly reduces the speed of movement.

Thus, instead of directly restoring material trajectories, it is proposed to implement the classification of trajectory elements (movement modes) from a previously known set. This approach avoids the accumulation of errors due to the recognition of modes and the subsequent formation of a new reference point. A similar approach is used in most modern physical activity trackers (fitness bracelets) [15-16].

In this case, a neural network is needed to classify fragments of time series. It is assumed that the records of the inertial unit will be fed sequentially to the neural network input. By processing the incoming measurements, a trained neural network will be able to determine (recognize) the mode of pelletizing charge in real time. Further, comparing the mode of pelletizing with the current technological parameters of the process (rotational speed of the drum, flow rate of the feed mixture and water for wet-

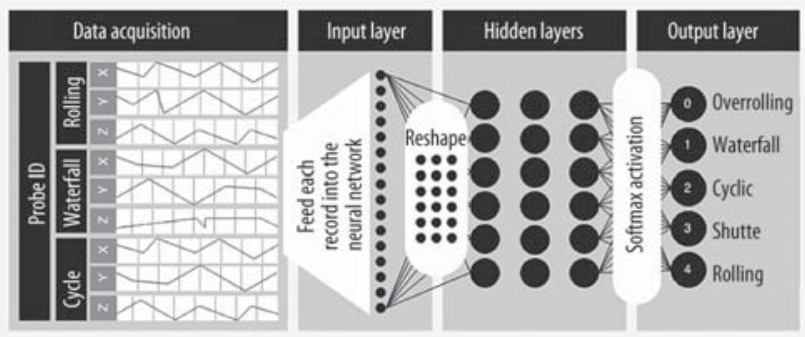

Fig. 2. Schematic representation of the deep neural network implementation

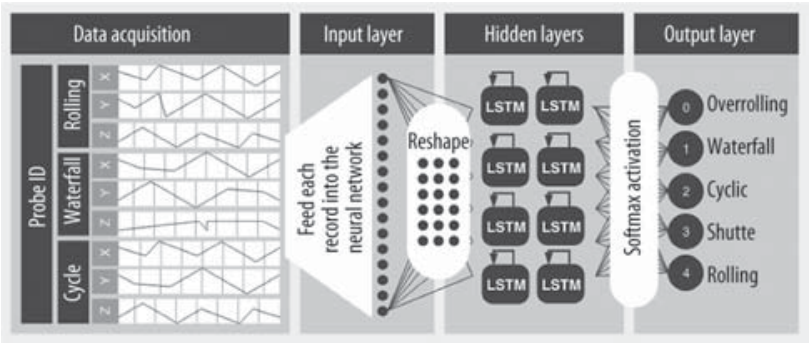

Fig. 3. Schematic representation of the LSTM implementation

ting), we can conclude about the current state of the pelletizing drum lining and the course of the whole process. To implement the neural network model, the open library TensorFlow from Google is used, in conjunction with the Keras framework.

Initially, a deep neural network (DNN) [17] model is tested (Fig. 2). The input data for the neural network are MEMS sensor accelerometers. The input layer of the neural network was a vector of 300 elements -100 measurements from each of the three accelerometers. Neural network has 3 hidden layers. The layers are completely interconnected. In front of the hidden layers there is an additional one that converts the vector of input data into a $100 \times 3$ matrix. In front of the output layer there is a fully connected one with the SoftMax activation function. The output layer consisted of 5 neurons. In each of layer the neural network returned the probability that the input vector belongs to one of the classes (modes).

As an alternative method, an architecture based on LSTM (Long short term memory) is considered. A feature of this architecture is its immunity to the duration of time gaps in the input data sequence, i.e., the ability of the neural network to memorize measurements for short periods of time. Due to this, the data obtained earlier did not significantly affect the further results of the operation of the neural network [18-19].

The neural network topology based on LSTM (Fig. 3) is identical as previously discussed. It had an input layer of 600 neurons, and in front of the output layer there is a layer with the SoftMax activation function. The main difference is in hidden layers.

To decide the suitability of the neural network model for use in a real information system, it is necessary to evaluate it. There is a very wide variety of metrics for 
assessing the quality of neural network models. In classification problems, the main metrics are accuracy and precision [20].

The simplest metric is the proportion of records by which the algorithm (neural network) correctly recognized the pelletization mode to the total number of records in the test sample. This metric is calculated by the equation (1):

$$
\text { Accuracy }=P / N \text {, }
$$

where $P$ - the number of records by which the pelletizing mode is correctly recognized; $N$ - total number of records in the sample.

The precision is a metric characterizing the number of all records that actually belong to a particular class, the sum of all instances that are assigned to this class by the algorithm. The metric is calculated using the following equation (2):

$$
\text { Precision }=T P / T P+F P,
$$

where $\mathrm{TP}$ - number of true positive answers; FP - number of false positives answers.

\section{Results and discussion}

An example of the algorithm work is shown in Fig. 4. It shows the conversion from the trajectory of a single particle when modeling the process of pelletizing into sensor readings (acceleration along each axis). Red indicates the movement of the tracker (particle) along a path that is indicated in black.

Then, the obtained accelerations were processed using the neural network model, which at the output gave the probability of belonging to one of the modes. In this

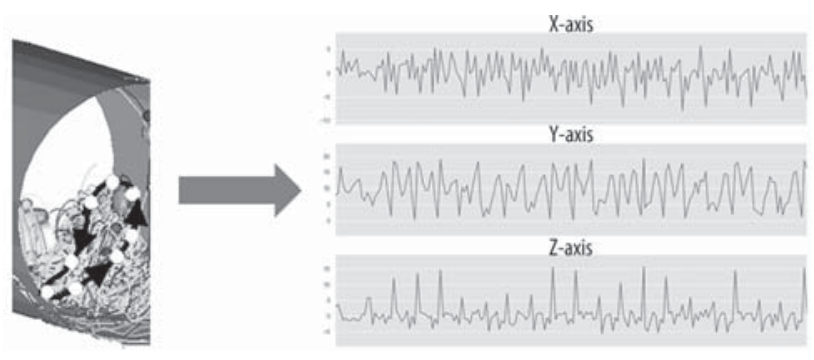

Fig. 4. Results of particle trajectory conversion into IMU readings

\begin{tabular}{|c|c|}
\hline \multicolumn{2}{|c|}{ Table 1. Distribution of modes among all trajectories } \\
\hline Pelletizing Mode & Percentage of total, \% \\
\hline Rolling & 8,9 \\
\hline Shuttle & 30,7 \\
\hline Overroling & 5,1 \\
\hline Waterfall & 3,9 \\
\hline Cyclic & 10,3 \\
\hline Jump & 41,1 \\
\hline
\end{tabular}

case, with a probability of $83 \%$, the pelletizing mode is Overrolling.

Among 10,981 trajectories, the most common mode is shuttle $-30.7 \%$ of the total. In addition, an auxiliary class is used to stop the flow analysis if the tracker left the device or did not yet hit it (Jump). Full details are given in table 1.

\subsection{Comparison of DNN and LSTM}

When evaluating the considered neural network classifiers according to these metrics, the following results are obtained: for a deep neural network (DNN), accuracy was $80 \%$, while precision $74 \%$. In other words, when DNN predicts a certain mode, then it is right in $74 \%$ of cases. Table 2 shows a matrix of confusion for a classifier based on a deep neural network.

As you can see from the matrix, DNN recognizes the shuttle mode and the jump well, but often confuses them. The remaining modes are recognized with varying success, which can be attributed to unsatisfactory recognition quality.

For networks of long-term short-term memory (LSTM), the accuracy was $96 \%$, precision $-97 \%$. This to a greater extent satisfies the necessary accuracy in determining the elements of the trajectories of the granular media movement in pelletizing drum. Table 3 shows the confusion matrix for the LSTM-based classifier.

In turn, the confusion matrix for LSTM is much better than DNN. Firstly, the diagonal elements of the matrix are clearly expressed - this is confirmed by a high precision of $97 \%$. Secondly, the number of recognition of classes that differ greatly along the trajectory (for example, rolling and shuttle modes) has significantly decreased. It is worth noting that a significant proportion of recognized classes are in jump and shuttle (about 70\%). Such a distribution is obtained due to a sample not ideally obtained from the simulation results. Ideally, the diagonal of the matrix should have approximately the same number of recognitions for each of the class. In addition, the possible recognition of very different modes during imple-

\begin{tabular}{c|c|c|c|c|c|c}
\hline \multicolumn{2}{l}{ Table 2. Confusion matrix for DNN } \\
\hline Overrolling & 175 & 35 & 0 & 0 & 213 & 227 \\
\hline Waterfall & 4 & 1883 & 0 & 0 & 32 & 71 \\
\hline Cyclic & 0 & 0 & 449 & 0 & 3 & 0 \\
\hline Shuttle & 0 & 0 & 93 & 272 & 5 & 0 \\
\hline Rolling & 69 & 35 & 5 & 27 & 306 & 283 \\
\hline Leap & 37 & 395 & 0 & 0 & 72 & 1893 \\
\hline & Overrolling & Waterfall & Cyclic & Shuttle & Rolling & Leap \\
\hline
\end{tabular}

\begin{tabular}{c|c|c|c|c|c|c}
\hline \multicolumn{7}{l}{ Table 3. Confusion matrix for LSTM } \\
\hline Overrolling & 975 & 3 & 0 & 0 & 68 & 12 \\
\hline Waterfall & 27 & 3370 & 0 & 0 & 44 & 6 \\
\hline Cyclic & 3 & 0 & 561 & 12 & 1 & 0 \\
\hline Shuttle & 7 & 0 & 1 & 427 & 1 & 1 \\
\hline Rolling & 50 & 2 & 0 & 1 & 1128 & 26 \\
\hline Leap & 24 & 7 & 0 & 0 & 18 & 4206 \\
\hline & Overrolling & Waterfall & Cyclic & Shuttle & Rolling & Leap \\
\hline
\end{tabular}


mentation introduces a fair question - how critical is it to make a decision about changing the process parameters based on an incorrectly recognized pelletizing mode? In general, this implies the need to improve the accuracy of class recognition, minimizing such errors. And with direct implementation in the early stages, use the system as an adviser, where the final decision is made by the operator.

\section{Conclusion}

Algorithms for recognizing the modes of bulk materials movement by the readings of an inertial measurement unit have been developed and implemented. The developed algorithms are based on neural network classifiers. Two types of classifiers are considered: on the basis of deep neural networks (DNN) and networks of long-term short-term memory (LSTM). The accuracy of recognition of trajectory elements by the DNN method was $80 \%$ in the test sample, which is associated with the influence of previous, largely remote in time, measurement results on the current state of the neural network. In the case of LSTM, the recognition accuracy of trajectory fragments in the training set was already $96 \%$, which satisfies the necessary accuracy in determining the elements of the motion paths of granular media in pelletizing drums for implementation as an adviser system.

The data on the nature of particle motion paths, which will be accumulated using the developed system, will make it possible to improve DEM models of the pelletizing process. Moreover, it is possible to create a methodology for improving DEM models of other technological processes on the basis of the system. For example, the methodology can be applied to study the movement of charge and grinding balls in a ball mill. The theoretical insights gained from studies of the models will be useful for improving and calibrating Rocky DEM's built-in fracture and particle interaction models (including friction coefficients).

The data on the elements of the particle motion paths accumulated using the developed device will make it possible to refine the DEM models of the pelletizing process and create a theoretical groundwork. On its basis, in the future, an automated pelletizing drum control system will be developed taking into account the variability of the properties of the charges fed to the unit. The nature of the bulk material movement in the process will also allow to assess the condition of the lining based on data on the frequency of sticking devices for the selected period.

Further work is to create a physical prototype of the device and implement it in real production. In addition, it is proposed to improve the algorithm for processing the readings of the built-in accelerometer to extract useful data. It is important to understand that the approach can be used not only in pelletizing machines, but also in other technological processes of processing and transport associated with bulk materials. The approach comes in line with the general trend for the digital technologies implementation at enterprises of the mineral sector.

\section{REFERENCES}

1. Sizyakov V. M., Vlasov A. A., Bazhin V. Yu. Strategic tasks of Russian metallurgical complex. Tsvetnye metally. 2016. No. 1. pp. 32-38.

2. Matveev I. A., Kalmykov A. V., Bespalov E. N. Compacting as an efficient technique for upgrading the grinding swarf and ensuring environmental safety during its storage, transportation and metallurgical processing. Tsvetnye metally. 2019. No. 5. pp. 74-80.

3. Golubev V. O., Chistyakov D. G., Brichkin V. N., Postika M. F. Population balance of aluminate solution decomposition. Tsvetnye metally. 2019. No. 8, pp. 75-81.

4. Gospodarikov A. P., Vykhodtsev Y. N., Zatsepin M. A. Mathematical modeling of seismic explosion waves impact on rock mass with a working. Journal of Mining Institute. 2017. Vol. 226. pp. 405-411.

5. Boikov A. V., Savelev R. V., Payor V. A., Erokhina O. O. The control method concept of bulk material behaviour in the pelletizing drum for improving the results of DEM-modeling. CIS Iron and Steel Review. 2019. Vol. 17. pp. 10-13.

6. Grishchenkova E.N. Development of a Neural Network for Earth Surface Deformation Prediction. Geotechnical and Geological Engineering. 2018. Vol. 36. No. 4. pp. 1953-1957.

7. Gogolinskiy K. V., Syasko V. A., Prospects and challenges of the fourth industrial revolution for instrument engineering and metrology in the field of non-destructive testing and condition monitoring. Insight. Non-Destructive Testing and Condition Monitoring. 2019. Vol. 61. No. 8. pp. 434-439.

8. Bui X. N., Nguyen H. Le H. A., Bui H. B., Do N. H. Prediction of Blast-induced Air Over-pressure in Open-Pit Mine: Assessment of Different Artificial Intelligence Techniques. Natural Resources Research. 2019. Vol. 29 No. 2. p. 571-591

9. Ahmad N. et al. Reviews on various inertial measurement unit (IMU) sensor applications. International Journal of Signal Processing Systems. 2013. Vol. 1. No. 2. pp. 256-262.

10. Kabanov E. I. Korshunov G. I., Gridina E. B. Algorithmic provisions for data processing under spatial analysis of risk of accidents at hazardous production facilities. Naukovyi Visnyk Natsionalnoho Hirnychoho Universytetu. 2019. Vol. 6. No. 6 pp. 117-121.

11. Hasan A. M. et al. Automatic estimation of inertial navigation system errors for global positioning system outage recovery. Proceedings of the Institution of Mechanical Engineers, Part G. Journal of Aerospace Engineering. 2011. Vol. 225. No. 1. pp. 86-96.

12. Brlow J. S. Inertial navigation as a basis for animal navigation. Journal of Theoretical Biology. 1964. Vol. 6. No. 1. pp. 76-117.

13. Goshen-Meskin D., Bar-Itzhack I. Y. Unified approach to inertial navigation system error modeling. Journal of Guidance, Control, and Dynamics. 1992. Vol. 15. No. 3. pp. 648-653.

14. Tikhonov A. A. Control Method for Angular Stabilization of an Electrodynamic Tether System. Automation and Remote Control. 2020. Vol. 81. No. 2. pp. 269-286.

15. Stauffer C., Grimson W. E. L. Learning patterns of activity using real-time tracking. IEEE Transactions on pattern analysis and machine intelligence. 2000. Vol. 22. No. 8. pp. 747-757.

16. Maung T. H. H. et al. Real-time hand tracking and gesture recognition system using neural networks. World Academy of Science, Engineering and Technology. 2009. Vol. 50. pp. 466-470.

17. Ciregan D., Meier U., Schmidhuber J. Multi-column deep neural networks for image classification. 2012 IEEE Conference on Computer Vision and Pattern Recognition, Providence, RI. 2012. pp. 3642-3649. DOI: 10.1109/CVPR.2012.6248110.

18. Graves A., Schmidhuber J. Framewise phoneme classification with bidirectional LSTM and other neural network architectures. Neural networks. 2005. Vol. 18. No. 5-6. pp. 602-610.

19. Mustafaev A. S., Sukhomlinov V. S. Analytical Theory of Energy Relaxation Upon Propagation of a High-Energy Electron Beam in Gas. High Temperature 2018. Vol. 56. No. 1. pp. 10-19.

20. Olden J. D., Joy M. K., Death R. G. An accurate comparison of methods for quantifying variable importance in artificial neural networks using simulated data. Ecological Modelling. 2004. Vol. 178. No. 3-4. pp. 389-397.

21. Kiangi K., Potapov A., Moys M. DEM validation of media shape effects on the load behaviour and power in a dry pilot mill. Minerals Engineering. 2013. Vol. 46-47. pp. 52-59. 